$16^{\text {th }}$ International Congress of Metrology, 03002 (2013)

DOI: $10.1051 /$ metrology/201303002

() Owned by the authors, published by EDP Sciences, 2013

\title{
Conceptual design of multi-capacity load cell
}

\author{
Seif. M. Osman ${ }^{1}$, Ebtisam H. Hasan ${ }^{1}$, H. M. El-Hakeem ${ }^{1}$, R.M.Rashad ${ }^{2}$ and F. Kouta ${ }^{2}$ \\ ${ }^{1}$ National Institute for Standards (NIS), Giza, Egypt. \\ ${ }^{2}$ Cairo University, Faculty of Engineering, Giza, Egypt.
}

\begin{abstract}
Force measuring systems (transducers and indicators) are usually used to calibrate force generated systems. Normally, it is not recommended to use load cells to measure forces less than $10 \%$ of its nominal capacity, thus, to offer calibration facilities for different ranges, several load cells are required, which may lead to difficulties in handling procedures in addition to the main disadvantage which is the over cost.

This article illustrates conceptual design for a new introduced multi-capacity load cell showing the design parameters and the required metrological characteristic of the new load cell.
\end{abstract}

\section{Force measurement systems}

A force measurement system is made up of a transducer and associated instrumentation. The transducer is subjected to the force to be measured, and the resulting change in the element is measured by the associated instrumentation. Force transducers can be distinguished accorduing to the type of output signal generated (mechanical, hydraulic, pneumatic or electrical).

\subsection{Mechanical Force Transducers.}

The most famous mechanical force transducer is the proving ring, where the deformation of the elastic element is measured via mechanical device. These transducers have the advantage of being simple and robust. The main disadvantage is the strong effect of temperature on the output.

\subsection{Piezoelectric Crystal load cells.}

These types differ from most other sensing elements in that they are active sensors. No power supply is needed and the deformation to generate a signal is very small. It had the advantage of a high frequency response of the measuring system without introducing geometric changes to the force measuring path. When a force is exerted on certain crystalline materials, electric charges are formed on the crystal surface in proportion to the rate of change of that force.

\subsection{Hydraulic Force Transducers.}

The hydraulic load cell is a device filled with a liquid -usually mercury-. It measure forces as a change in pressure of the internal filling fluid. The force acting on its loading head is transferred to a piston that in turn compresses a filling fluid confined within an electrometric diaphragm chamber. As force increases, the pressure of the hydraulic fluid rises. This pressure can be measured by a pressure transducer or displayed on a pressure gauge dial via a Bourdon tube or escape in a pipe and measure via a micrometer. [1].

\subsection{Electrical Force Transducers. ( load cell)}

Basically, a load cell consists of specially designed structures which perform in a predictable and repeatable manner when a force is applied. The force applied is translated into a voltage by the resistance change in strain gages which are intimately bonded to the transducer structure.

Wheatstone bridge circuit is ideal for measuring the resistance changes that occur in strain gages, normally, the strain gages are connected in a four arm Wheatstone Bridge configuration fig(1), which acts as an adding and subtracting electrical network and allows for compensation for temperature effects.

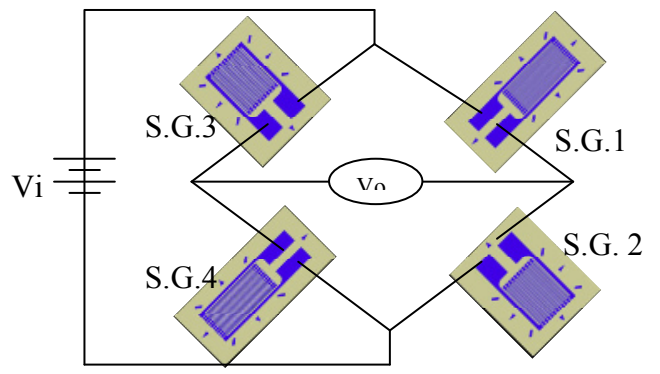

Fig(1), Wheatstone bridge

The rated capacities of strain gauge load cells range from a few Newton to $30 \mathrm{MN}$. They have become the most widespread of all force measurement systems. [2].

\section{Load cells loading principle}

Load cells have different principle, it can be based on bending, shear or axial loading.

\subsection{Bending principle.}

Bending principle could be single or double. Single bending load cell is designed to be bolted to a support

\footnotetext{
Seif M. Osman: SeifElnasr_nis@yahoo
} 
through the two mounting holes. There is a large hole bored through the beam. This forms thin sections at the top and bottom surface, which concentrate the forces into the area where the gauges are mounted on the top and/or bottom faces of the beam. The gauges may be mounted on the outside or inside surface of the large hole.

Double Bending Beam Cell, shown in figure 2, is consider a development for the single beam theory. It is achieved by forming two bending beams into one cell. This allows the loading fixtures to be attached at the threaded holes on the center line, between the beams, which makes the sensitive axis pass through the cell on a single line of action. In general, this configuration is much more user friendly [3].

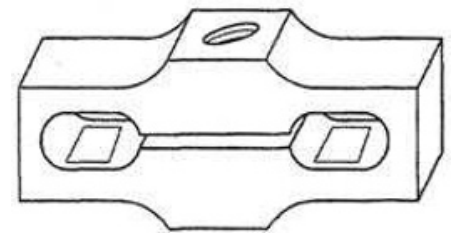

Figure 2. Double-ended beam

\subsection{Shear principle.}

This principle looks identical to a bending beam cell, Howeve, the large hole differ that in the bending beam, instead of passing all the way through the cell, there is a thin web in the center of the cell and the gauge is oriented at $45^{\circ}$. Thus the force on the end of the beam causes the web to be stressed in shear, which has a maximum effect at $45^{\circ}$. Fig. (3). [3]

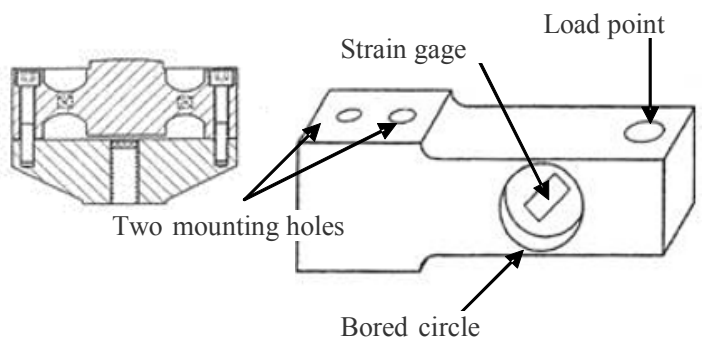

Fig (3) Shear beam flexure

\subsection{Axial loading principle.}

It is the simplest form of the elastic element. The elastic element is a column, which is loaded axially either in tension or compression. Normally, the elastic element has massive blocks at the top and bottom and a thin, usually square, column in the center.

\section{The Multi-capacity load cell}

A new concept for designing a load cell with multi capacity is very important and an indeed need, to cover different ranges of loads. This concept is an innovation in force measurement field as it hasn't been discussed before. The strain gauge load cell is the most common commercially available type of force transducer and is recommended to be used in this multi-capacity load cell. The strain gage is the heart of the load cell.

\subsection{Concepts to be considered when designing multi-capacity load cell:}

\subsubsection{Loading direction}

Load cells generally are designed for measurement in either tension or compression but some universal load cells are designed for measurement in both directions. Load cells work in compression modes are simpler in design than others.

\subsubsection{Loading nature (static or dynamic)}

Loading speed and how fast the reading is $(1 \mathrm{~Hz}$, $10 \mathrm{~Hz}, 100 \mathrm{~Hz}$ or faster) are the main factors in determining the loading nature either static or dynamic. The design of load cells differs according to the required response. The response time of a load cell is generally of the order of 3-5 milliseconds.

Loading nature also determines the type of required output; Analog or Digital. The most common output available from conventional load cells is $\mathrm{mV} / \mathrm{V}$.

\subsubsection{Size}

Dimensions are the controlling consideration in applications. Comfort of users, ease of use, safe handling and monitoring conditions to prevent harmful faults are to be considered when designing load cells . A special attention should be paid to he loading mounting accessories of load cells on the top as well as bottom for compression and tension modes. The main purpose of these mounting accessories is to ensure axial, accurate and safe loading conditions.

\subsubsection{Characteristics of elastic element}

The shape of the elastic element on which the strain gages are bonded depends on a number of factors including the range of force to be measured, dimensional limits, final performance and production costs. The force carrying member may be a column or tension bar of circular, square or octagonal cross section and may be either solid or hollow. The member can also be of ring or other form designed to produce a simultaneous measurement by the gauges.

The material used for the elastic element is usually a material which exhibits a linear relationship between the stress (force applied) and strain (output) with low hysteresis and low creep in the working range. There also has to be high level of repeatability between force cycles to ensure that the load cell is a reliable measuring device. To achieve these characteristics it is usual to subject the material to a special heat treatment [4].

The geometric shape and modulus of elasticity of the elastic element determine the magnitude of the strain field produced by the action of the force.

Building a multi capacity load cell require using different elastic element for each range. The multi capacity load cell is based on increasing the stiffness for each range.

Stiffness $k$ is the resistance of an elastic body to deformation by an applied force. It is determined from the equation: 


$$
\text { Stiffness }=\frac{\text { Load }}{\text { Deformation }} \text { or } k=\frac{P}{\Delta L}
$$

Where: $\mathrm{k}$ is the stiffness, $\mathrm{P}$ is the load and $\Delta \mathrm{L}$ is the deformation

\section{- Stiffness relationship to elasticity}

In general, elastic modulus is not the same as stiffness. Elastic modulus is a property of the constituent material and the stiffness is a property of a structure. Stiffness depends on the material, shape and boundary conditions. They are related by:

$$
E=\frac{\sigma}{\varepsilon}, \sigma=\frac{P}{A}, \varepsilon=\frac{\Delta L}{L}, k=\frac{P}{\Delta L}=\frac{\sigma A}{\varepsilon L}=\frac{E A}{L}
$$

Where: $\mathrm{E}$ is the modulus of elasticity, $\sigma$ is the material strength , $\varepsilon$ is the material strain , A is the cross-sectional area and $\mathrm{L}$ is the initial length

The previous relation shows that, the modulus of elasticity is often one of the primary properties considered when selecting a material. A high modulus of elasticity is sought when deflection is undesirable, while a low modulus of elasticity is required when flexibility is needed. Stiffness can be determined for each material by relating it with the material property.

Simply in the first range of the multi capacity load cell an elastic element nominated for the working range is loaded. For the next range either a new element is loaded separately - without any loading on the first element- or the new introduced element is loaded with the first element, hence the two elements withstand the load.

In case of a new element is added in parallel, the new stiffness is calculated from:

$$
C=C_{1}+C_{2}
$$

If the new element is added in series the stiffness is calculated from:

$$
\frac{1}{C}=\frac{1}{C_{1}}+\frac{1}{C_{2}}
$$

Using more than one element in series decreases the stiffness while using more than one element in parallel increases the stiffness, which is recommended in the multi-capacity load cell.

\subsubsection{Accuracy}

Accuracies of load cells are generally rated as a percentage of full scale capacity.

\subsubsection{Environmental conditions.}

The environmental conditions (temperature and humidity) where the designed load cell will be used must be accurately determined and taken in considerations during design phases as it could affect the load cell accuracy and sensitivity. It is more commercial to design load cells which offer certain degree of temperature compensation.

\section{- Temperature effects on strain.}

The change in temperature $\Delta \mathrm{T}$ is related to the thermal normal strain $\varepsilon_{\mathrm{t}} . \quad \varepsilon_{t}=\alpha \Delta T$, where $\alpha$ is the linear coefficient of thermal expansion.

The stress-strain curve is affected by the increase in temperature [5], as the temperature increase before the application of the force, the stress-strain curve starting point moves from point $\mathbf{O}$ to point $\mathbf{O}_{1}$, as shown in Figure 4. The total strain at any point is the sum of mechanical strain and thermal strains, which can be written as follows: $\varepsilon=\frac{\sigma}{E}+\alpha \Delta T$

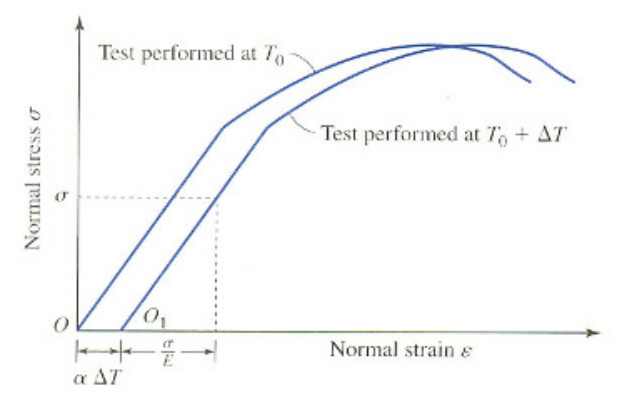

Fig. (4) temperature effect on strain

\section{- Temperature Compensation in the Bridge Circuit}

Temperature variations mainly affect the zero point. Errors can be maintained within reasonable bounds by the selection of self-compensating strain gauges and/or the use of suitable compensation methods.

Strain gauge specifications are determined at a reference temperature (usually $23^{\circ} \mathrm{c}$ ). The gauge factor is one of the specifications which change with temperature change, this correlation is linear and the temperature coefficient of the gauge factor and its tolerance are stated on each strain gage package. Strain gauges must be selected according to the thermal expansion coefficient $\alpha$ of the test material. They are manufactured to compensate the thermal effect of temperature change on the elastic element material.

Wheatstone bridge circuit is used to carry out compensation. The ability to make such compensation is one of the more desirable features of this circuit. This is accomplished as follows.

The extraneous effects of temperature and other factors inducing a resistance change in the gage can effectively be considered as an additional strain, $\varepsilon_{\mathrm{i}}{ }^{\mathrm{t}}$ so that the strain in the i-th arm becomes $\varepsilon+\varepsilon_{i}^{t}$

Then the full bridge equation can be written [6]: $e=\frac{G F}{4}\left[\varepsilon_{1}-\varepsilon_{2}+\varepsilon_{3}-\varepsilon_{4}\right] E+\frac{G F}{4}\left[\varepsilon_{1}^{t}-\varepsilon_{2}^{t}+\varepsilon_{3}^{t}-\varepsilon_{4}^{t}\right] E$ Where the term $\frac{G F}{4}\left[\varepsilon_{1}^{t}-\varepsilon_{2}^{t}+\varepsilon_{3}^{t}-\varepsilon_{4}^{t}\right] E$ represents the extraneous temperature effects. 
When similar strain gauges are used in all four arms of the bridge and when they are mounted so that each experience the same temperature change, then the bridge output voltage will be a function of the material strain only. In this case the temperature induced resistance changes will be the same for each gauge and their contribution to the bridge output will cancel.

\subsubsection{Safety}

To use a load cell safely, it is recommended not to increase the load over 1.5 times its rated maximum capacity.

\section{Conclusion.}

This new concept is a new facility in force measurements and can be considered an innovation in force measurement field as it is introduced for the first time in this article.

This concept can be used to design and manufacture a multi-capacity load cell that provides high precise measurement for different ranges. The previous suggested designs show the need of high accurate manufacturing techniques in order to have an accurate, compact, handled load cell covering more than one range safely and with relatively low cost

\section{Future work}

The research group decides to propose an effective design of the multi-capacity load cell based on the design concepts introduced in this article. This will be the first load cell to carry out this work.

\section{References}

1-Wolpert Werkstoffprufmacschinen, Amsler calibrating boxes MD31, Company pub.1.52/31.

2-Karl Hoffmann, "An Introduction to Measurments using Strain Gages", Hottinger Baldwin Messtechnik GmbH, Darmstadt, 1989.

3-http://www.interfaceforce.com/technicallibrary/load_cell_intro4.php. $14^{\text {th }}$ February, 2012..

4-Dan Mihai ${ }^{-}$Stefanescu, Alexandru Stefanescu."Criteria for Choosing the Elastic Elements of Force Transducers" Proceedings of the $17^{\text {th }}$ International conference IMEKO TC3. (Sept.2001). pp134-140

5-R.K. Rajput "Materials Science And Engineering", S.K. Kataria \& sons, third edition 2006, ISBN:81-85749-92-2.

6-http://soliton.ae.gatech.edu/people/jcraig/classes/ae3 145/Lab2/strain-gages.pdf. 19th September. 2012 